\title{
Influência da embalagem na aceitação de diferentes marcas comerciais de cerveja tipo Pilsen
}

\author{
Influence of packaging on the acceptability of different commercial brands of Pilsen beer
}

\author{
Milene Moreira RIBEIRO ${ }^{1}$, Suzana Maria DELLA LUCIA ${ }^{1}$, Paula Bucharles Franco BARBOSA ${ }^{1}$,

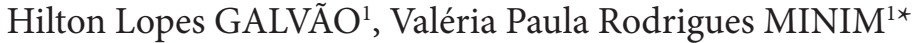

\begin{abstract}
Resumo
Foram estudadas nove marcas de cervejas comerciais brasileiras tipo Pilsen, com o objetivo de avaliar a influência da embalagem na aceitação das mesmas. As amostras foram separadas em três grupos e baseadas na pesquisa Top of Mind/2005. As marcas 1, 2, 3 e 4 (grupo a) foram as mais citadas pelos consumidores nacionais; as 5 e 6 (grupo b) tiveram citação intermediária; e as 7, 8 e 9 (grupo c) são consideradas regionais. O trabalho foi dividido em 3 etapas: teste cego, teste com embalagem e teste com informação. Os testes foram aplicados a 54 julgadores, em condições laboratoriais, usando escala hedônica de 9 categorias. Os resultados foram analisados por ANOVA e teste de Tukey (p $\leq 0,05)$. No teste cego, a marca 1 diferiu significativamente das marcas 4, 7 e 8, sendo a menos aceita. No teste com embalagem, a situação se inverteu, sendo a marca 1 a mais aceita, juntamente com as marcas 3, 4 e 9, e a marca 7 foi a menos aceita. Já no teste com informação, as cervejas do grupo a e a marca 8 (regional) foram as mais aceitas. Os resultados indicam que muitas vezes a embalagem influencia e pode modificar a aceitação de algumas cervejas.
\end{abstract}

Palavras-chave: marca; teste cego; análise sensorial; informação.

\begin{abstract}
Nine Brazilian commercial brands of Pilsen beer were studied to evaluate the influence of packaging on their acceptability. The brands were separated into three groups, based on the Top of Mind/2005 research. Brands number 1, 2, 3 and 4 (group a) are the most recognized by national consumers; brands number 5 and 6 (group b) have intermediate recognition and brands number 7, 8 and 9 (group c) are considered regional. This study was separated in 3 stages: blind test, test with labels and test with information. The tests were applied to 54 consumers under laboratory conditions, using a nine-point hedonic scale. The results were analyzed using ANOVA and the Tukey test ( $\mathrm{p} \leq 0,05)$. In the blind test, brand 1 was significantly different from brands 4,7 and 8 , being the less accepted. In the test with labels, the situation was inverted, since brand 1 was the most accepted, along with brands 3, 4 and 9; and brand 7 was the less accepted. On the other hand, in the test with information, Group A beers and brand 8, which is regional, were the most accepted. The results indicate that the labels can often influence and modify the acceptability of some beers.
\end{abstract}

Keywords: brand; blind test; sensory analysis; information.

\section{Introdução}

Quando se fala em consumo nacional de bebidas, a cerveja está em segundo lugar no ranking, perdendo apenas para os refrigerantes (CERVEJA, 2006).

"Mania nacional", a cerveja é a bebida alcoólica mais consumida no Brasil. O país é o quinto maior produtor e consumidor de cerveja, em termos de volume. Segundo o SINDICERV (Sindicato Nacional da Indústria da Cerveja) (SINDICATO, 2006), o consumo da bebida, em 2005, apresentou crescimento de aproximadamente $6,5 \%$ em relação ao ano anterior, atingindo o patamar de 90 milhões de hectolitros/ano. Quanto ao consumo per capita, houve um aumento de 46,6 L em 2004 para 49 L em 2005 por habitante.

A cerveja é uma das bebidas mais delicadas e lábeis, possuindo um complexo, mas moderado, e aroma e sabor que são atributos sensoriais responsáveis pela aceitação e qualidade da bebida (CERVEJA, 2006). A cerveja do tipo Pilsen nasceu em
Pils, na ex-Tchecoslováquia, em 1842, e é a mais conhecida e consumida no mundo. De sabor delicado, leve, clara e de baixo teor alcoólico (entre 3 e 5\%), é também a preferida dos brasileiros. No Brasil, o consumo da Pilsen chega a 98\% do total, ficando o restante para as cervejas do tipo Bock, Light, Malzebier e Stout (SINDICATO, 2006).

No entanto, a escolha do consumidor é influenciada por outras informações e atributos não sensoriais, tais como: identificação do produto, situação de compra e consumo, experiências anteriores com o produto, origem, segurança e propriedades nutricionais, além da marca e hábitos pessoais do consumidor (ARAÚJO; SILVA; MINIM, 2003; DRANSFIELD; ZAMORA; BAYLE, 1998; SIRET; ISSANCHOU, 2000; TUORILA; CARDELLO; LESHER, 1994).

Como a qualidade é um instrumento fundamental para se obter vantagens no mercado competitivo, pois ela influencia

${ }^{1}$ Departamento de Tecnologia de Alimentos - DTA, Universidade Federal de Viçosa - UFV, Campus Universitário, CEP 36571-000, Viçosa - MG, Brasil, E-mail: vprm@ufv.br ${ }^{*}$ A quem a correspondência deve ser enviada 
o comportamento do consumidor, é necessário ter informações acerca do produto, de modo que este possa satisfazer as necessidades e expectativas do consumidor (CAPORALE; MONTELEONE, 2004), adequando-se aos padrões de qualidade ditados por ele. É nesse contexto que se torna interessante pesquisar as percepções e os anseios do cliente a quem o produto é direcionado.

O estudo do comportamento do consumidor é tarefa multidisciplinar, envolvendo ciência e tecnologia de alimentos, nutrição, psicologia e marketing (GUINARD; UOTANI; SCHLICH, 2001). A percepção das características de produtos alimentares demonstrou ser afetada por muitos fatores individuais, que incluem atributos sensoriais que interagem com fatores fisiológicos, comportamentais e cognitivos dentro da experiência do consumidor, influenciando sua percepção. $O$ contexto e as experiências também afetam essa interação (LANGE; ROUSSEAU; ISSANCHOU, 1999).

Dentre esses fatores, as expectativas com relação ao produto possuem importante papel, uma vez que elas podem melhorar ou piorar a percepção do mesmo, antes de ele ser experimentado (DELIZA; MACFIE, 1994).

A expectativa pode ser gerada por atributos externos e não sensoriais, tais como: informação do produto (SIRET; ISSANCHOU, 2000), informação nutricional (DELIZA; MACFIE, 1994) preço (DI MONACO et al., 2004), embalagem e rótulo (SMYTHE; BAMFORTH, 2002). A embalagem pode levar o consumidor a comprar o produto, enquanto as características sensoriais confirmam a apreciação e podem determinar a reincidência na compra (MURRAY; DELAHUNTY, 2000).

A marca é considerada muito útil quando os consumidores estão escolhendo entre produtos competitivos. Durante o processo de compra, os consumidores buscam informações da memória e do ambiente externo e processam e armazenam os resultados de sua compra em sua memória, para que estes sejam usados em outras compras similares. A presença de uma marca bem estabelecida no mercado, portanto, é uma forte influência na formulação das expectativas sensoriais dos consumidores, assim como em seu comportamento de escolha e compra e na sua aceitação (DELIZA; MACFIE, 1994; DI MONACO et al., 2004; JAEGER, 2006).

O presente trabalho teve por objetivo avaliar a aceitação de diferentes marcas comerciais nacionais de cerveja do tipo Pilsen a partir de seus atributos sensoriais e da combinação destes com informações fornecidas na sua embalagem.

\section{Material e métodos}

\subsection{Amostras}

Nove marcas de cervejas nacionais do tipo Pilsen, em embalagens de lata, disponíveis no mercado de Viçosa - MG foram adquiridas para os testes sensoriais. As diferentes marcas foram reunidas em três grupos ( $\mathrm{a}, \mathrm{b}$ e c ), de acordo com dados da pesquisa Top of Mind/2005 - categoria Cerveja (TOP, 2006), no que se refere à lembrança destas pelos consumidores. As marcas 1, 2, 3 e 4 (grupo a) representavam empresas líderes de mercado, com cerca de $75 \%$ das menções, as marcas 5 e 6 (grupo b) representavam empresas de porte médio (cerca de $8 \%$ de menções para cada marca) e as marcas 7, 8 e 9 (grupo c) totalizavam menos de $1 \%$ do total de lembranças, podendo ser consideradas regionais.

\subsection{Testes de aceitação}

Foram recrutados 54 voluntários para compor a equipe sensorial, entre estudantes e funcionários da Universidade Federal de Viçosa (UFV). O recrutamento foi realizado por meio de questionário relativo a dados demográficos dos participantes, disponibilidade de tempo e freqüência de consumo do produto.

Os testes de aceitação foram conduzidos no Laboratório de Análise Sensorial da UFV, em três sessões, com intervalos de dois dias entre cada sessão. As amostras (bebida e embalagem) foram codificadas com três dígitos, estes alterados em cada dia de avaliação para evitar que o julgador fosse influenciado pela sessão anterior.

Na primeira sessão (teste cego) os consumidores degustaram aproximadamente $50 \mathrm{~mL}$ da bebida, servida em taças de cristal (capacidade de $50 \mathrm{~mL}$ ), sem obter informação sobre qual marca de cerveja estava em avaliação.

Na segunda sessão (teste da embalagem) foi avaliada a aceitação das embalagens das amostras servidas na sessão anterior. Esse procedimento permitiu que o consumidor avaliasse, entre outros atributos, a marca das cervejas em estudo.

A avaliação das amostras de cerveja servidas juntamente com a respectiva embalagem foi realizada na terceira sessão (teste com informação), na qual foi solicitado ao consumidor que julgasse a bebida, atentando para o fato de que esta era proveniente do produto contido naquela embalagem.

As análises foram realizadas em cabines individuais e as amostras servidas de forma aleatória e monádica, em temperatura de refrigeração $\left( \pm 7^{\circ} \mathrm{C}\right)$ em cada sessão, sob luz branca. Uma vez que o número de amostras em avaliação era grande para a análise pelo julgador (nove amostras de cerveja), em cada sessão houve um intervalo de aproximadamente 15 minutos entre a avaliação das cinco primeiras amostras e das quatro últimas. Este intervalo evitou que o julgador viesse a ter fadiga sensorial por degustar seguidamente várias amostras. Em todas as condições de teste o julgador recebeu uma ficha para cada amostra, em que lhe foi solicitado que indicasse na escala hedônica de 9 pontos (Figura 1) o seu julgamento em relação à aceitação do produto ou da embalagem, atribuindo nota 9 para "gostei extremamente" e 1 para "desgostei extremamente" (MINIM, 2006).

\subsection{Análise estatística}

A análise de variância $(p \leq 0,05)$ e o teste de Tukey foram realizados para a comparação das médias, ao se estudar as marcas dentro de cada sessão e no estudo comparativo da aceitação das amostras entre as sessões (LANGE; ROUSSEAU; ISSANCHOU, 1999). 
As análises estatísticas foram feitas utilizando-se o programa Statistical Analysis System (SAS), licenciado para uso pela UFV.

\section{Resultados e discussão}

Dos 54 julgadores selecionados para compor a equipe sensorial, $80 \%$ eram homens e $20 \%$ mulheres; $96 \%$ eram estudantes de graduação ou pós-graduação da UFV.

A análise de variância apresentou diferença significativa entre as médias de aceitação das marcas quando estas foram avaliadas no teste cego, conforme ilustrado na Figura 2.

As médias de aceitação variaram entre 5,2 (marca 1) e 6,6 (marca 8), situando-se entre os termos hedônicos "indiferente" e "gostei moderadamente". Observou-se que os julgadores

Teste de aceitação

Nome:

Sexo: Idade:

Por favor, avalie a amostra utilizando a escala abaixo para dizer o quanto você gostou ou desgostou do produto. Marque a posição que melhor reflita o seu julgamento.

Código da amostra:

( ) gostei extremamente

( ) gostei muito

( ) gostei moderadamente

( ) gostei ligeiramente

( ) indiferente

( ) desgostei ligeiramente

( ) desgostei moderadamente

( ) desgostei muito

( ) desgostei extremamente

Comentários:

Figura 1. Modelo de ficha utilizada para a avaliação da aceitação das amostras de cerveja e das respectivas embalagens.

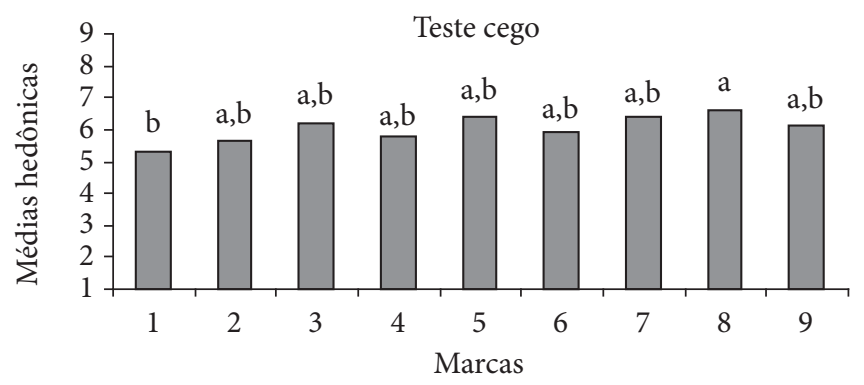

Figura 2. Médias de aceitação para as nove marcas de cerveja testadas na sessão 1 (teste cego). Médias com a mesma letra não diferem entre si pelo teste de Tukey $(\mathrm{p}>0,05)$. posicionaram suas preferências na parte média da escala. Isto sugere que estes se mostraram inibidos ao julgar as amostras devido à ausência de identificação das mesmas, não explorando, portanto, toda a amplitude da escala, como observado também por Di Monaco et al. (2004).

A marca 1, uma das empresas líderes de mercado, foi menos aceita que a marca 8, uma das marcas de cerveja regionais.

No segundo teste, o qual avaliou a influência da embalagem, a análise de variância aplicada às médias de aceitação das marcas obtidas revelou, assim como para o teste cego, diferença entre as amostras (Figura 3).

Neste teste as médias variaram entre 5 (marca 7 ) e 7,2 (marca 3). As diferenças entre as médias de aceitação das amostras foram um pouco mais pronunciadas do que nas condições de teste cego (Figura 2).

As marcas 3, 1, 2 e 5 tiveram aceitação similar. A marca 8 não diferiu de 1, 2 e 5. A marca 7 foi a que teve menor aceitação, mas não se diferenciou das marcas 4, 6 ou 9. Observa-se que, para as marcas 1, 2 e 3, o fornecimento da informação sobre o produto (contida na embalagem) foi positivo. Isso corrobora o fato de que a familiarização com essas marcas ou o conhecimento dos consumidores acerca de tais produtos permitiu haver influência nas avaliações, no momento em que as informações sobre o produto foram fornecidas durante a análise sensorial (ARRUDA et al., 2006; DELLA LUCIA et al., 2006; DI MONACO et al., 2004; GUINARD; UOTANI; SCHLICH, 2001). Trabalhos anteriores demonstraram que o maior volume de informação fornecido ao consumidor em relação ao produto influencia sua avaliação sensorial; o fornecimento de informações relacionadas não somente à marca, mas também à tecnologia de plantio (JOHANSSON et al., 1999) e fabricação do alimento (CAPORALE; MONTELEONE, 2004), e a origem do mesmo (CAPORALE; MONTELEONE, 2001) tende a modificar o comportamento do consumidor no momento da escolha e do consumo do produto.

Della Lucia et al. (2006) constataram também a mudança no comportamento do julgador ao estudarem a expectativa gerada pela embalagem sobre diferentes marcas de iogurte; as líderes de mercado obtiveram médias inferiores ao serem degustadas, em comparação aos resultados obtidos com relação às respectivas embalagens. Assim, mesmo não sendo tão aceita

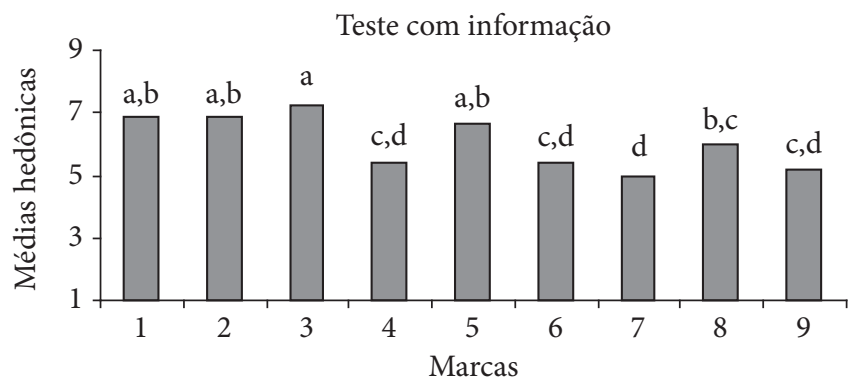

Figura 3. Médias de aceitação para as nove marcas de cerveja testadas na sessão 3 (teste com informação). Médias com a mesma letra não diferem entre si pelo teste de Tukey $(\mathrm{p}>0,05)$. 
ao ser degustada, a amostra conhecida tende ser a preferida quando se conhecem suas características, ou seja, quando estas são fornecidas. O mesmo comportamento foi observado em consumidores de cerveja norte-americanos perante diferentes marcas e preços (GUINARD; OUTANI; SCHLICH, 2001).

Analisando as marcas por grupos, constata-se que três marcas do grupo a, juntamente com a marca 5 (grupo b), foram mais aceitas que as demais. Quanto às amostras do grupo c, observouse que a marca 8 foi relativamente bem aceita (Figura 3); esta apesar de se enquadrar no grupo $c$, de acordo com o Top of Mind (2006), é muito conhecida no mercado de Viçosa -MG e talvez por este fato tenha tido médias de aceitação superiores. Para as marcas do grupo b, empresas de porte médio em relação ao consumo, a aceitação não variou em função do fornecimento da informação ao julgador.

Quando a marca é informada, ela gera uma distorção nas respostas dos consumidores para algumas amostras. A Figura 4 representa um paralelo entre as médias de aceitação das três sessões para as nove marcas de cerveja.

É interessante perceber que as notas para as marcas de cerveja do grupo a mudaram em geral para uma posição mais alta, à medida que se fornecia informação ao consumidor acerca das amostras. Em contrapartida, as notas para as marcas 7 e 9 mostraram-se inferiores nesta mesma situação.

Para algumas marcas, o fornecimento de informação, em função da exposição das embalagens, influenciou negativamente a aceitação da bebida. Este fato sugere uma visão pejorativa do consumidor perante algumas marcas, principalmente aquelas de mercado regional. Isto se deve, em grande parte, ao fato de que o investimento em marketing para produtos regionais ou de menor mercado, em empresas de menor porte, é inferior àquele dado em grandes empresas, que geralmente desenvolvem estratégias mercadológicas mais direcionadas e que causam maior impacto positivo sobre o consumidor.

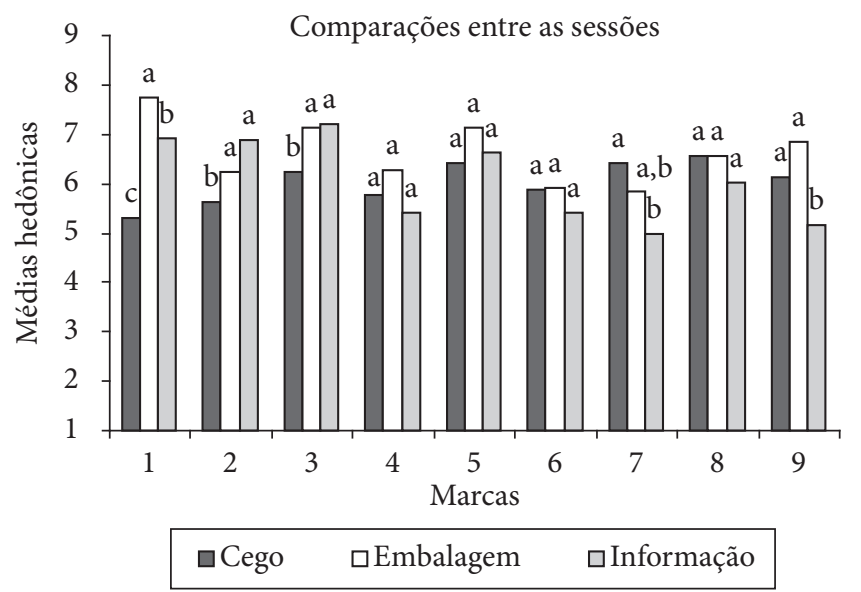

Figura 4. Médias de aceitação para as nove marcas de cerveja testadas nas sessões 1 (teste cego), 2 (teste da embalagem) e 3 (teste com informação). Médias com a mesma letra não diferem entre si pelo teste de Tukey $(\mathrm{p}>0,05)$.
Dentro das estratégias de marketing, o trabalho de crescimento e desenvolvimento de uma marca é um fator primordial para o sucesso do mercado. A marca do produto é, na maioria das vezes, decisiva para o processo de escolha e compra. A marca conhecida ou desconhecida exerce influência considerável sobre a expectativa do consumidor, razão pela qual este atributo tem sido pesquisado com tanta freqüência (DELLA LUCIA et al., 2006; DI MONACO et al., 2004; DRANSFIELD; ZAMORA; BAYLE, 1998; GUINARD; OUTANI; SCHLICH, 2001) como uma informação determinante para o cliente. Uma marca conhecida tende a expressar maior confiança ao consumidor.

Fica fácil visualizar a influência da informação contida na embalagem sobre a aceitação do consumidor. A familiaridade com a marca de cerveja, refletida no fornecimento da informação nas sessões 2 e 3, gerou maior aceitação das amostras familiares, apesar de as mesmas nem sempre terem obtido maior média no teste com amostras codificadas (teste cego).

\section{Conclusões}

Os resultados indicaram que a familiaridade com algumas marcas de cerveja afetou a aceitação do consumidor, refletida pelas maiores médias obtidas para sua embalagem, já que os julgadores modificaram sua aceitação quando a embalagem foi fornecida. Algumas marcas influenciaram de maneira negativa a aceitação da bebida, demonstrando efeito pejorativo sobre a avaliação do consumidor.

A falta de conhecimento da marca do produto gerou insegurança nos provadores quanto à exposição de seus julgamentos, durante o teste cego. Com isto, houve uma tendência central das respostas, ou seja, os consumidores posicionaram suas respostas entre os pontos 5 e 7 da escala hedônica.

Observa-se, portanto, neste estudo como é marcante a influência da embalagem na aceitação dos produtos e fica evidente que o investimento em marketing é um fator determinante na conquista do mercado.

\section{Agradecimentos}

Ao $\mathrm{CNPq}$, pelo suporte financeiro.

\section{Referências bibliográficas}

ARAÚJO, F. B.; SILVA, P. H. A.; MINIM, V. P. R. Perfil sensorial e composição físico-química de cervejas provenientes de dois segmentos do mercado brasileiro. Ciência e Tecnologia de Alimentos, Campinas, v. 23, n. 2, p. 121-128, 2003.

ARRUDA, A. C. et al. Cafés convencional, orgânico e descafeinado: impacto da informação na sua aceitação. Revista Brasileira de Armazenamento, Especial Café, Viçosa, n. 9, p. 94-99, 2006.

CAPORALE, G.; MONTELEONE, E. Effect of expectations induced by information on origin and its guarantee on the acceptability of a traditional food: olive oil. Sciences des Aliments, v. 21, p. 243-254, 2001.

Influence of information about manufacturing process on beer acceptability. Food Quality and Preference., v. 15, n. 3, p. $271-278,2004$. 
Cerveja: um mercado em expansão. Disponível em: <http://www. bndespar.gov.br/conhecimento/bnset/cerveja.pdf\#search= \%22cerveja\%20consumo\%22>. Acesso em: 09 set. 2006.

DELIZA, R.; MACFIE, H. J. H. The generation of sensory expectations by external cues and its affects on sensory perception and hedonic ratings: A review. Journal of Sensory Studies, v. 11, p. 103-128, 1994.

DELLA LUCIA, S. M. et al. Expectativa gerada pela embalagem sobre a aceitabilidade de iogurte sabor morango. In: Congresso Nacional de Laticínios, 23, 2006, Juiz de Fora-MG. Anais... Juiz de Fora - MG: Revista do Instituto de Laticínios “Cândido Tostes”, v. 61, n. 351, p. 1-429, jul/ago, 2006. (CD-ROM).

DI MONACO, R. et al. The effect of expectations generated by brand name on the acceptability of dried semolina pasta. Food Quality and Preference, v. 15, n. 5, p. 429-437, 2004.

DRANSFIELD, E.; ZAMORA, F.; BAYLE, M. C. Consumer selections of steaks as influenced by information and price index. Food Quality and Preference, v. 9, n. 5, p. 321-326, 1998.

GUINARD, J. X.; UOTANI, B.; SCHLICH, P. Internal and external mapping of preferences for commercial lager beers: comparison of hedonic ratings by consumers blind versus with knowledge of brand and price. Food Quality and Preference, v. 12, n. 4, p. 243-255, 2001.

JAEGER, S. R. Non-sensory factors in sensory science research. Food Quality and Preference, v. 17, n. 1-2, p. 132-144, 2006.

JOHANSSON, L. et al. Preference for tomatoes, affected by sensory attributes and information about growth conditions. Food Quality and Preference, v. 10, n. 4-5, p. 289-298, 1999.
LANGE, C.; ROUSSEAU, F.; ISSANCHOU, S. Expectation, linking and purchase behavior under economical constraint. Food Quality and Preference, v. 10, n. 1, p. 31-39, 1999.

MINIM, V. P. R. Análise sensorial: estudos com consumidores. Viçosa: Editora. UFV, 225p, 2006.

MURRAY, J. M.; DELAHUNTY, C. M. Mapping consumer preference for the sensory and packaging attributes of Cheddar cheese. Food Quality and Preference, v. 11, n. 5, p. 419-435, 2000.

SINDIVERV - Sindicato Nacional da Indústria da Cerveja. Mercado. 2002/2003. Disponível em: <http://www.sindicerv.com.br/mercado. php>. Acesso em: 09 set. 2006.

SIRET, F.; ISSANCHOU, S. Traditional process: influence on sensory properties and on consumers' expectation and liking. Application to paté de campagne. Food Quality and Preference, v. 11, n. 3, p. 217-228, 2000.

SMYTHE, J. E.; BAMFORTH, C. W. A study of the effect of perceived beer history on reported preferences by sensory panels with different levels of training. Journal of the Institute of Brewing, v. 108, n. 1, p. 34-36, 2002.

TOP OF MIND - As marcas campeãs 2006. Apresentação 2005Alimentação-Cerveja. Disponível em: $<$ http://datafolha.folha.uol. com.br/produtos/top_index.shtml\#>. Acesso em: 09 set. 2006.

TUORILA, H.; CARDELLO, A. V.; LESHER, L. L. Antecedents and consequences of expectations related to fat-free and regular-fat foods. Appetite, v. 23, n. 3, p. 247-263, 1994.

WESTCOMBE, A.; WARDLE, J. Influence of relative fat content information on responses to three foods. Appetite, v. 28, n. 1, p. 49-62, 1997. 